Ciencia y Educación, Vol. 4, No. 2, mayo-agosto, 2020

ISSN (impreso): 2613-8794・ISSN (en línea): 2613-8808

DOI: https://doi.org/10.22206/cyed.2020.v4i2.pp7-25

\title{
Dificultades del profesorado en sus funciones docentes y posibles soluciones. Un estudio descriptivo actualizado
}

\author{
Diffculties of teachers in their teaching functions and possible solutions. \\ An updated descriptive study
}

\author{
Francisco José Rubio Hernández ${ }^{a}$ ORCID: 0000-0001-7736-912X \\ José Luis Olivo-Franco ${ }^{b}$ ORCID: 0000-0002-7781-1261
}

Recibido: 27/12/2019 • Aprobado: 12/02/2020

Cómo citar: Rubio Hernández, F. J., \& Olivo-Franco, J. L. (2020). Dificultades del profesorado en sus funciones docentes y posibles soluciones. Un estudio descriptivo actualizado. Ciencia y Educación, 4(2), 7-25. Doi: https://doi.org/10.22206/cyed.2020. v4i2.pp7-25

\section{Resumen}

Los obstáculos a los que tienen que hacer frente los docentes actuales deben ser reevaluados en función de la sociedad contemporánea, cambiante y de la inmediatez. El objetivo de esta indagación fue describir las dificultades en las funciones docentes de una muestra de 31 profesionales y las posibles soluciones para mejorar su praxis. Se ejecutó una investigación cuantitativa, no experimental, trasversal y descriptiva. Los problemas prioritarios de estos docentes fueron la sobrecarga de trabajo, la motivación del alumnado, el mantenimiento de la disciplina y la burocracia. Entre las propuestas de mejora destacaron potenciar la valoración de lo actitudinal-emocional en los estudiantes, fomentar las relaciones entre el profesorado-familia e impulsar el desarrollo de estrategias de enseñanza-aprendizaje. Se concluye advirtiendo sobre la detección de algunos cambios respecto a las dificultades de los enseñantes, y la necesidad de explorar otras variables, como pueden ser las del ámbito de las TIC y su impacto en dicho asunto.

Palabras clave: docente de escuela primaria; docente de secundaria; docencia.

\begin{abstract}
The obstacles that current teachers have to face must be re-evaluated based on a contemporary changing society and immediacy. The objective of this investigation was to describe the difficulties in the teaching functions of a sample of 31 professionals and the possible solutions to improve their praxis. A quantitative, non-experimental, cross-sectional and descriptive research was carried out. The priority problems of these teachers were: work overload, student motivation, maintaining discipline, and bureaucracy. Among the improvement proposals, they highlighted promoting the assessment of the attitudinal-emotional aspects of the students, promoting relationships between teacher-family and promoting the development of teaching-learning strategies. It concludes by warning about the detection of some changes regarding the difficulties of teachers, and the need to explore other variables, such as those in the field of ICT and its impact on this matter.
\end{abstract}

Keywords: Primary school teacher; secondary school teacher; teaching.

\footnotetext{
a Departamento de Métodos de Investigación y Diagnóstico en Educación. Facultad de Educación. Universidad de Murcia, España. Correo-e: frubio@um.es

b Institución Técnica Agrícola “Juan Domínguez Romero". Malambo, Atlántico, Colombia.

Correo-e: joseolivofranci@hotmail.com
} 


\section{Introducción}

La educación es, tal como apunta Zayas, Gonzálves y García (2019), uno de los mayores retos que enfrentan las sociedades con miras a promover transformaciones. Así pues, son precisamente los docentes sobre quienes recae el liderazgo que promueva tales cambios.

No obstante, las dificultades a las que debe hacer frente el profesorado en sus funciones docentes, ya sea desde la perspectiva del profesor experimentado como del que inicia su profesión, han sido estudiadas desde hace varias décadas (Cardona, 2008; Flores, 2015; Imbernón, 1998; Marcelo, 1991, 1999; Veenman, 1984). Sin embargo, la sociedad es cambiante, el alumnado y los demás actores que conforman el entramado educativo evolucionan. De hecho, dichas transformaciones sociales, económicas y culturales afectan a los centros educativos y, en consecuencia, a los docentes, los cuales se encuentran profundamente tensionados por los roles que tradicionalmente desempeñaban y por otros que la ciudadanía les exige (Sánchez-Sánchez y Jara-Amigo, 2019). Por tanto, es pertinente indagar de nuevo para comparar los datos de antańo con los actuales, ya que, como señala Montero y Gewerc (2018), las dificultades a las que debe hacer frente el cuerpo docente son una cuestión perdurable en educación.

Como se sabe, en el ámbito escolar han surgido nuevas demandas y expectativas, las cuales se han planteado a partir de los cambios sociales, económicos y culturales que han tenido lugar en muy diversos terrenos (Day, 2005). Así, desde el primer decenio del siglo xxI, la diversidad cultural en España ha aumentado considerablemente, la inclusión y desarrollo de las Tecnologías de la Información y la Comunicación se ha convertido en tal fenómeno que los estudiantes avanzan a más velocidad que los docentes en su uso y manejo, produciéndose interrogantes en la necesidad de cambios en la forma de afrontar la clase (Roig, Mengual y Quinto, 2014; Tejada y Pozos, 2018).

Ante este escenario cambiante surgen nuevos desafíos que constituyen a su vez nuevas dificultades, así como múltiples situaciones éticas y morales en la labor de los docentes de hoy, quienes demandan nuevas compe- tencias y saberes, que merecen ser abordados a través de estudios que posibiliten una mejor aproximación a tal problemática, con miras a que hayan una mayor claridad acerca de qué debe saber un o una docente para enseñar, cómo enseñarlo, cómo debe saberlo para aprender (Cardona, 2013, Franco-López, López-Arellano y Arango-Botero, 2020; Moliner y Ortí, 2016) y cómo hacer frente a tales obstáculos para desarrollar su profesión con unos mínimos garantes de calidad (Álvarez-Álvarez, 2015).

\section{Marco teórico}

La praxis docente requiere, tal como lo plantean Jiménez, Jara y Miranda (2012), un alto compromiso motivacional y, al mismo tiempo, señalan que es una profesión de alto riesgo, puesto que los docentes deben estar preparados para enfrentar diversas situaciones, como la desmotivación de los estudiantes (cada vez más extendida), reclamos y presiones de los padres y madres de los estudiantes, la escases de apoyo de la institución donde ejercen, la ausencia de recursos, la planificación, coordinación y evaluación en una escuela cada vez más turbada (Franco-López et al., 2020; Vázquez-Cano, 2016). Realidades inéditas y complejas como las nuevas tecnologías, la variedad de tipos y de estructuras familiares (Abril y Peinado, 2018), así como "las nuevas formas de violencia escolar, la inmigración o educación en contextos vulnerables" (Martín-Romera y García-Ramírez, 2018, p. 8). Todas ellas constituyen escenarios que redefinen la profesión docente, tal como lo expresan Martín-Romera y García-Ramírez (2018).

Ahora bien, usualmente en la literatura se pueden revisar investigaciones sobre y con el profesorado. Las indagaciones sobre el profesorado han vertido datos e informaciones en torno a elementos relacionados con la desprofesionalización, modelos formativos de los docentes, diferencias entre profesorado principiante y experimentado, entre otros aspectos. Mientras que las segundas, las investigaciones "con" han permitido desarrollar nuevos procesos de las prácticas educativas, formativas, comunicativas, el desarrollo del profesor como investigador y los procesos de formación en las instituciones educativas. 
Teniendo en cuenta que el saber docente puede clasificarse en cuatro grupos, atendiendo a "la planificación, la toma de decisiones, las teorías o creencias, y la diferencia entre profesorado novato o experto" (Ros-Garrido y Chisvet-Tarazona, 2018, p. 97), también es posible encontrar estudios que aborden las dificultades del magisterio desde estas cuatro categorías.

Sin embargo, y sobre este tópico, la mayoría de investigaciones se han desarrollado atendiendo al criterio de profesorados noveles o con experiencia, clara tendencia seguida por la generalidad de estudios acerca de este tema. Así, por ejemplo, se pueden citar autores como Abril y Peinado (2018), Álvarez-Álvarez (2015), Cardona (2008), Eirín, García y Montero (2009a), Flores (2015), Marcelo (2008), Moliner y Ortí (2016), cuyas investigaciones siguen esta línea. Ahora bien, no existe una definición única de profesor novel y no existe consenso sobre en cuántos años de labor se supera esta condición (Moliner y Ortí, 2016). No obstante, vale la pena seńalar que no siendo el caso que ocupa al presente trabajo, no se dará mayor relevancia a este tópico o variable.

Con respecto a las dificultades del profesorado, se han categorizado de diferentes formas, como ya se ha dicho, obedeciendo generalmente a la distinción entre docentes noveles o con experiencia. De esta manera, es posible referenciar diversas dificultades, tales como problemas que obstaculizan las relaciones entre la teoría y la práctica señalados por Álvarez-Álvarez (2015); dificultades relacionadas con la dimensión académica, como motivar y explicar lecciones al alumnado, tratamiento del alumnado en forma diferenciada e individualizada, entre otros (Moliner y Ortí, 2016); dificultades para planificar, coordinar y evaluar (Vásquez-Cano, 2016), o dificultades en las dimensiones organizativas; dificultades de índole social, dificultades en el manejo uso y aplicación de los materiales-tecnológicos (Moliner y Ortí, 2016). Con respecto a esto último vale decir que los profesores y las profesoras del siglo Xxi deben tener como eje de su formación inicial, avanzada y continua la comprensión y estudio de su teoría y práctica educativa para desde la formación investigativa producir saber pedagógico y didáctico desde su contexto (Tovar-Gálvez, 2018).
No obstante, un referente clásico obligado a la hora de considerar el tópico en cuestión es el estudio de Veeman (1988). Básicamente, todos los estudios posteriores coinciden con este, el cual señala dificultades del profesorado categorizadas en diferentes ámbitos, tales como:

La disciplina en el aula, la motivación de los alumnos, su organización del trabajo en clase, la sobrecarga laboral, la falta de tiempo para preparar las clases, el dominio de diferentes métodos de enseñanza y de la materia, la determinación del nivel de aprendizaje de los alumnos, los insuficientes e inadecuados materiales didácticos, las relaciones con los padres y con los otros profesores, el trabajo burocrático, las relaciones con los directivos, la falta de tiempo libre, la falta de apoyo y orientación y, por último, el número de alumnos por aula. (Flores, 2015, p. 415)

Asimismo, dentro de los problemas que debe enfrentar el profesorado cabe destacar la intensificación del trabajo docente, definido desde las teorías generadas del proceso de trabajo. Según apuntan Hargreaves (2005) y posteriormente Sánchez y Andrade (2014), los planteamientos de dicha teoría pueden resumirse en tres afirmaciones que han sido sintetizadas en la figura 1 .

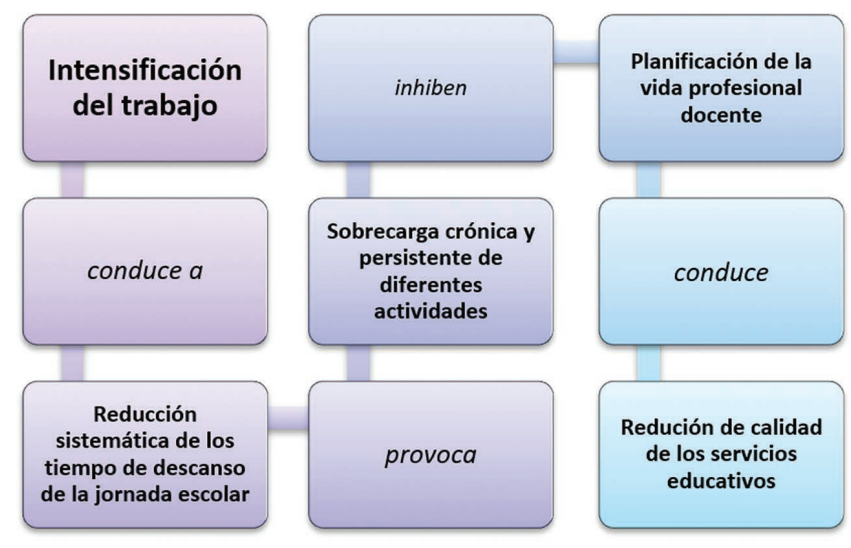

Figura 1. Planteamientos principales de la teoría de Hargreaves sobre la intensificación del trabajo

Fuente: elaboración propia a partir de Hargreaves (2005). 
Por otro lado, hay que reconocer que la naturaleza del saber docente se caracteriza por la pluralidad y complejidad, también por una continua evolución y cambio. Ejemplo de esto es que actualmente las necesidades del alumnado no son las mismas que hace un lustro, ni tampoco simétricas a las de hace una o varias décadas. Estas nuevas necesidades que los estudiantes presentan exigen al profesorado buscar, encontrar, crear, adaptar y/o probar formas de enseñar innovadoras. Así, será necesaria la detección, selección y eliminación de los conocimientos profesionales caducos. También la capacidad de apoyar o renovar aquellos válidos hoy en día. Por tanto, es esta nueva forma de enseñar que tiene que aprender el docente lo que va a determinar, como ya advertía Cardona (2008b), la esencia y propiedades del saber docente.

En cuanto a la labor que desempeńa un profesor o una profesora, se debe atender a las diferencias que existen entre la función docente tradicional (la enseñanza) y las funciones docentes actuales del profesorado, las cuales trascienden la de antaño, pues acumula otras muchas que la sociedad les exige, debido, sobremanera, a las múltiples nuevas necesidades que los estudiantes y sus familias han experimentado. En esta línea, el profesorado se ha visto obligado a introducir innovaciones en su praxis, tales como los modelos colaborativos de actuación, la multiplicación del conocimiento y de los caminos de acceso al mismo, las nuevas formas de atender a los conflictos entre escolares (Rubio, Díaz y Cerezo, 2019) y mantener la disciplina en el aula, así como la diversificación de sus tareas. Todo esto ha ido perfilando lo que hoy día es la labor del docente.

Por ende, la sociedad posmoderna empuja a plantear una nueva forma ser docente, con características nuevas y otras renovadas y cuyo rol ya no es el mismo. Entre esos desafíos se encuentran los de atender a estratos sociales en riesgo (la crisis económica ha aumentado los índices de pobreza infantil, así como la reducción de recursos en miles de familias), la diversidad de culturas en los centros escolares, la introducción de las TICs en la vida cotidiana de las personas (Roig et al., 2014), pero también, por parte del alumnado, una pérdida del sentido del saber y de aprender, lo que trae consigo la desmotivación de niños y adolescentes. Para dar respuesta a esta diversidad de nuevas situaciones, los maestros y las maestras deben considerar su oficio como una constante de aprendizaje y, sin duda, los Estados apostar y aumentar su inversión en educación.

De esta forma, como se atisbó anteriormente, existe una clara relación entre cambios sociales, culturales y económicos en relación con lo educativo. Por ello, las necesidades sociales actuales demandan una modificación, un cambio o trasformación no solo en las funciones del profesorado, sino además en su rol. Ambos, funciones y rol, deben estar teñidos de innovación desde la cual abordar su formación inicial y continua (Cardona, 2008b).

Una de estas transformaciones se evidencia en la relación docente-estudiante y que en la modernidad pierde vigencia debido, entre otros aspectos, al aprieto por el que pasa el profesor o profesora como figura de autoridad educativa. También, por los cambios que han sufrido las instituciones educativas (Alfaro, 2011).

Por consiguiente, y en relación con el rol docente actual, se descarta la consideración de un maestro o maestra como única fuente de acceso y de transmisión del conocimiento; esto es, como persona que transfiere información linealmente de los conocimientos que sabe a un estudiante pasivo que los recibe sin más (rol del docente tradicional). Hoy en día se avanza hacia un nuevo rol de profesor que se implica en una nueva forma de organizar y entender la enseńanza y el aprendizaje, que actúa como guía y también como facilitador del aprendizaje del estudiante.

En consecuencia, el docente debe adquirir un nivel avanzado en el dominio (y uso crítico y deontológico también) de las Tecnologías de la Información y la Comunicación (Sánchez, Boix y Jurado, 2009), desarrollar una capacidad que le permita ser generador de contenidos que se adapten a dichas herramientas y que, además, se acoplen y sean adecuadas a su metodología en el proceso de enseñanza-aprendizaje. De esta manera, volviendo al rol de profesor 
como un facilitador, mediador y guía de los aprendizajes, es él quien, como enseñante también, presenta a su alumnado modos de búsqueda y diferentes usos que pueden dar a la información de forma eficiente, distinguiendo la fiable y valiosa de la que no lo es (Molas y Rosselló, 2010).

Respecto a las características del docente actual, Cardona (2008b), a partir de lo propuesto por Montero (1999), considera que los elementos que caracterizan al profesor de hoy, análogamente con el dinamismo histórico del proceso de su profesionalización, son los que exponemos a continuación: a) su autonomía profesional, es decir, la capacidad del profesional de la educación de controlar sus decisiones y autogobernar su profesión. En contraposición, esa autonomía profesional decrece en el ámbito de la colectividad, en la toma de decisiones del centro escolar en su conjunto; b) su aislamiento profesional: por aquello de que cada aula es un espacio reducido o mínimo separado de las demás, cada docente trabaja de forma autónoma, sin apenas compartir experiencias o buenas praxis con sus compañeros para aprender y mejorar; c) la feminización del cuerpo profesional, debido a un mayor número de mujeres docentes, sobre todo en las etapas de Educación Infantil y Primaria; d) una carrera profesional rasa y uniforme, donde la tónica predominante es la insuficiencia de vías de progreso dentro del mismo cuerpo y la escasa promoción interna; e) los riesgos psicológicos de la profesión del docente, dado además de, por las anteriores características, por otras, tales como la crisis de autoridad, la poca consideración social de su oficio, la incidencia de los fenómenos de acoso escolar y ciberacoso (Cerezo y Rubio, 2017), problemas para los que el profesorado cuenta con una formación escasa o nula (Rubio, Díaz y Cerezo, 2019), así como otras dificultades en la convivencia que surgen de sus relaciones con los entornos próximos al centro y sus actores (aula, escuela, familias, comunidad), lo cual contribuye al sufrimiento de estrés y ansiedad por parte de los docentes (Sánchez, 2007).

Tras repasar el papel que desempeña el docente en su actividad (su rol), las cualidades que determinan qué rasgos le son propios al docente (sus características), es el momento de abordar cuáles son las actividades que desarrolla el profesorado en el desempeño de su oficio, es decir, la función (funciones) del docente contemporáneo. De esta forma, siguiendo a Cardona (2008b), se puede considerar la función docente como las "actividades, acciones, misiones o tareas que realizan unas personas a quienes se les reconocen conocimientos, habilidades y actitudes, así como valores, adecuados para enseñar a las personas" (Cardona, 2008b, p. 157).

Por ello, parece pertinente exponer las siete dimensiones operativas que recogen algunas de las tareas definitorias de la función docente, atendiendo a Cardona (2008b): a) dimensión planificadora (dentro de esta se encuentra el diagnóstico de necesidades del alumnado, la preparación de clases o sesiones, el diseño de entornos TIC de aprendizaje o la búsqueda y preparación de recursos y materiales didácticos relacionados con la materia); b) dimensión metodológica (entre cuyas tareas se encuentra la orientación a los estudiantes hacia el aprendizaje autónomo, la motivación del alumnado en su proceso de aprendizaje, el desarrollo de una docencia que parta de la diversidad del alumnado y de sus necesidades e intereses, la constitución del docente como una de las fuente de información para alumnos y alumnas, el fomento del autoaprendizaje y de la participación, entre otras); c) dimensión asesora y tutorial; d) dimensión evaluadora; e) dimensión modélica (es decir, actuar como ejemplo para el alumnado); f) dimensión investigadora y desarrollo profesional (donde cabría incluir la experimentación en el aula, la realización de trabajos con el alumnado, la valoración de los resultados obtenidos por estos, así como la formación continua del profesorado); g) dimensión gestora.

Por otro lado, retomando las dificultades a las que deben hacer frente los profesores y profesoras en sus funciones docentes, se recoge ahora una propuesta en la figura 2 que aborda los obstáculos a los que deben enfrentarse al inicio de su carrera. 


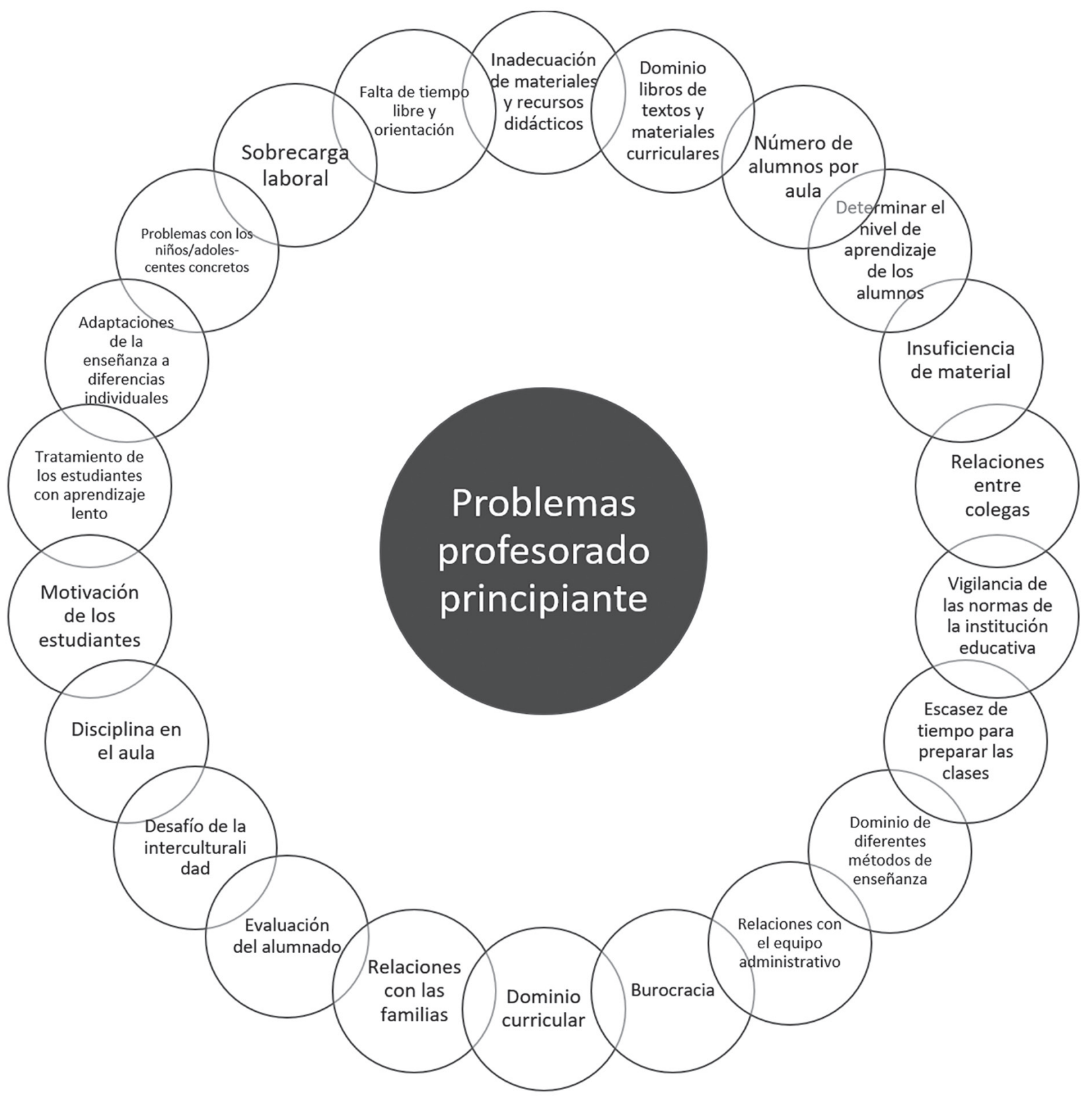

Figura 2. Principales obstáculos a las que se enfrenta el profesorado al inicio de su carrera Fuente: elaboración propia a partir de Imbernón (1998)

De modo similar, Perrenoud (2011) recoge los rasgos que caracterizan al docente novel y que permiten dilucidar algunas dificultades a las que se puede enfrentar el profesorado, siendo destacadas las siguientes: 1) crisis de identidad debido al paso de estudiante a profesional; 2) el estrés, la angustia y el miedo que disminuyen con la experiencia y la autoconfianza; 3) el desgaste de concentración y tiempo para afrontar satisfactoriamente las diferentes problemáticas es mayor que los docentes con experticia; 4) su gestión del tiempo es poco eficaz y eficiente, lo cual le genera estrés; 5) estado de sobrecarga cognitiva, con una cantidad excesiva de problemas, experimentan la angustia de la dispersión; 6) soledad y dificultades para integrarse con el resto del grupo de trabajo, sumado a que ocasionalmente no son bien 
acogidos; 7) dificultad para separar su rol de las situaciones problemáticas; y 8) autopercepción de que no domina con eficacia las acciones propias de su profesión.

Por otro lado, en Educación Primaria Obligatoria, las principales dificultades son la falta de información sobre los estudiantes y su contexto y ambiente familiar (también los problemas de disciplina). En cuanto al profesorado que atiende al alumnado con necesidades específicas de apoyo educativo, las preocupaciones docentes enfatizan el número de estudiantes desproporcionado por clase o sesión. Asimismo, sobre el escaso conocimiento del estudiante como ser (Espino, Galván, y Blanco, 2019).

En consecuencia, y con el objetivo de ayudar y facilitar ese camino que va de la facultad (donde uno es estudiante) hasta el colegio o instituto (donde comienza a ejercer de docente), se crearon los programas de iniciación a la docencia (Eirín et al., 2009). Estos programas persiguen mejorar el aprendizaje de la enseñanza en los futuros docentes, introducir y después retener a los profesores principiantes, así como revitalizar a los docentes con experiencia a través del desempeño de roles nuevos, esta vez como mentores. Con todo esto se busca mejorar tanto la eficacia profesional del cuerpo docente como su autoestima (Moliner y Ortí, 2016).

Por otra parte, para el profesorado principiante son estos programas de inducción una oportunidad que le permite crecer profesionalmente, pues se le brinda la ocasión de observar y aprender buenas prácticas educativas (y su posterior implementación), recibir ayuda, orientación y asesoramiento por parte de otros compañeros y compañeras más experimentados, quienes transfieren sus conocimientos y prácticas a los recién llegados al oficio de profesor.

Por añadidura, además de lo anterior, se ha apuntado en diferentes estudios la eficacia de participar en los grupos de apoyo para docentes principiantes, en los cuales es posible la compartición de experiencias, materiales y recursos educativos, las dificultades y problemas de la práctica diaria, las estrategias de actuación e intervención, lo que hace que el nuevo docente se sienta en un ambiente más libre, relajado e informal que en el de continua evaluación por parte de los compañeros más experimentados, que, a veces, se percibe como una relación jerárquica y caracterizada por la superioridad-inferioridad (Eirín, García y Montero, 2009b; Cardona, 2013). Parece pues interesante y adecuado optar por ambas actuaciones, ya que posibilitan al docente novel aprender de forma global y transitando por diferentes tipos de conocimientos, estrategias y prácticas.

Análogamente con lo precedente, siguiendo a Marcelo (2008), es preciso derribar la contradicción que reconoce que para mejorar la calidad de la educación son indispensables los docentes, pero que, por otro lado, las instituciones y políticos muestran escaso interés e inversiones en al ámbito educativo y menos en el de la formación y desarrollo docente.

Todas estas aportaciones, algunas de hace una o dos décadas y otras más recientes, han llegado, en muchas ocasiones, a conclusiones caracterizadas por su similitud (Cardona, 2013), aunque se debe tener presente que la sociedad de hace dos decenios, por ejemplo, poco tiene que ver con la actual, en la que está presente la multiculturalidad, una escuela que se esfuerza por ser cada vez más inclusiva y superar la integración, lo cual denota falta de equidad educativa. Debido a esto y a otras variables expuestas en párrafos anteriores, surge la necesidad de proceder a una evaluación de la problemática actual de los docentes.

\section{Preguntas de investigación y objetivos}

Se plantean las siguientes preguntas de investigación: ¿qué dificultades presentan los profesores y profesoras en sus funciones docentes y cuáles son las soluciones que proponen para solventarlas? ¿Las dificultades y soluciones detectadas son similares o diferentes a las propuestas por investigaciones previas?

Por tanto, el objetivo general es describir las dificultades en las funciones docentes de una muestra de profesores y profesoras de la Comunidad Autónoma de la Región de Murcia (España), así como las posibles soluciones para mejorar su praxis.

Este propósito general se concreta en los siguientes objetivos específicos: a) describir las dificultades en relación a las funciones docentes señaladas por el profesorado en general; b) especificar las soluciones más importantes para mejorar el desempeño de las funciones docentes del profesorado. 


\section{Metodología}

\section{Diseño}

Atendiendo a la clasificación de Van Dalen (1990), García y Martínez (2012), así como de Hernández-Sampieri y Mendoza (2018), el diseño de la investigación fue cuantitativo, no experimental, transeccional o transversal y descriptivo. Con esta elección se pretendió obtener información para detectar dificultades y soluciones, describirlas y, tras ello, proponer cambios que puedan implicar mejoras.

\section{Participantes}

La población objeto de estudio la conformaron las profesoras y profesores de los centros educativos de una localidad de la Comunidad Autónoma de la Región de Murcia, España. En concreto un colegio público de educación infantil y educación primaria, así como un instituto de educación secundaria.

Para conseguir la muestra, se empleó una técnica de muestreo no aleatorio por conveniencia. Treinta y un (31) sujetos formaron parte de la misma: nueve (9) hombres y veintidós (22) mujeres. Las características de la muestra, atendiendo a diferentes estratos (sexo, etapa educativa y grado de experiencia), pueden visualizarse en la tabla 1.

Tabla 1. Distribución por estratos de los participantes

\begin{tabular}{|c|c|c|c|c|c|c|}
\hline & $\begin{array}{c}\text { Hom- } \\
\text { bres }\end{array}$ & $\begin{array}{l}\text { Muje- } \\
\text { res }\end{array}$ & Total & $\begin{array}{c}\text { Experi- } \\
\text { mentados }\end{array}$ & Noveles & Total \\
\hline $\begin{array}{c}\text { Educación } \\
\text { infantil }\end{array}$ & 1 & 5 & 6 & 5 & 1 & 6 \\
\hline $\begin{array}{c}\text { Educación } \\
\text { primaria }\end{array}$ & 3 & 11 & 14 & 9 & 5 & 14 \\
\hline $\begin{array}{l}\text { Educación } \\
\text { secundaria }\end{array}$ & 5 & 6 & 11 & 11 & 0 & 11 \\
\hline Total & $\begin{array}{c}9 \\
(29 \%)\end{array}$ & $\begin{array}{c}22 \\
(71 \%)\end{array}$ & $\begin{array}{c}31 \\
(100 \%)\end{array}$ & $\begin{array}{c}25 \\
(80,6 \%)\end{array}$ & $\begin{array}{c}6 \\
(19,4 \%)\end{array}$ & $\begin{array}{c}31 \\
(29 \%)\end{array}$ \\
\hline
\end{tabular}

Fuente: elaboración propia

\section{Variables}

En la investigación se contemplaron variables sociodemográficas y variables relacionadas con: a) problemas en el desempeño de las funciones docentes del profesorado (dificultades a las que los profesoras y profe- sores deben hacer frente en su centro educativo, aula, en relación con las familias, etcétera); b) iniciativas para mejorar el desempeño de las funciones docentes del profesorado (son propuestas que se podrían tener en cuenta para incorporar en los grados y postgrados universitarios en educación, en la formación continua del profesorado, en las políticas educativas y en los modos de organización de los centros educativos, entre otros menesteres).

Las variables sociodemográficas, con sus correspondientes opciones de respuesta, son las siguientes: edad (respuesta abierta), sexo (hombre, mujer), años de experiencia docente (respuesta abierta), nombre del centro (respuesta abierta), etapa educativa (educación infantil, educación primaria, educación secundaria obligatoria, educación secundaria postobligatoria), tipo de centro (público, privado, privado-concertado), localidad (respuesta abierta), país (respuesta abierta).

El resto de variables presentan las siguientes opciones de respuesta: muy en desacuerdo/nada importante, en desacuerdo/poco importante, ni de acuerdo ni en desacuerdo/regular, de acuerdo/importante, muy de acuerdo/muy importante.

Se aprecia, por tanto, la inclusión de variables cualitativas nominales (dicotómicas y politómicas) y variables cualitativas ordinales o cuasicuantitativas, además de las que admiten respuestas abiertas.

\section{Instrumentos}

El instrumento de diagnóstico socioeducativo empleado para la recogida de datos e información fue el cuestionario de Cardona (2008). Dicho instrumento se compone de seis (6) dimensiones constituidas por 83 ítems. A través de ellos se recoge de forma general y específica las opiniones y valoraciones de los docentes de niveles no universitarios sobre ciertos aspectos relacionados con la problemática en su ejercicio, así como algunas posibles estrategias para paliar o superar dichas dificultades.

Además de la revisión bibliográfica realizada por el autor señalado, la validez de contenido se llevó a cabo mediante el procedimiento interjueces, concretamente por catorce (14) expertos en las temáticas. Por otra parte, el análisis de fiabilidad se ejecutó mediante el cálculo del alfa de Cronbach. Todas las dimensiones 
arrojaron un alfa superior a $.70(.95, .80, .86, .78$, $.81, .90)$, lo cual se considera como aceptable.

No obstante, para este estudio se analizaron los resultados de la primera, cuarta, quinta y sexta dimensión.

\section{Procedimiento}

Se contactó telefónicamente con los centros educativos para invitar a sus docentes a participar en la investigación. Una vez aceptaron, se estableció una planificación para la aplicación de los cuestionarios a los profesores y profesoras. La recogida de datos e información tuvo lugar en seis (6) jornadas, en horario de mañana, concretamente durante el receso, para así poder contactar más fácilmente con los sujetos. Tras ello, se procedió al análisis de datos y a la presentación e interpretación de los resultados.

En otro orden de cosas, se debe señalar que a los docentes no se les proporcionó consentimiento informado por escrito para que lo firmasen, pero sí de forma oral. Se entregó un documento donde se les informaba del objetivo de la indagación y una breve descripción de la misma. También acerca de que el estudio era voluntario y las respuestas anónimas y confidenciales. Los sujetos no percibieron incentivos por su participación. Uno de los investigadores supervisó la administración de los instrumentos de medida.

Por otro lado, la investigación no recibió aprobación de ningún comité ético científico universitario, aunque sí se llevó a cabo siguiendo los principios de la Declaración Helsinki, adaptado al ámbito de las ciencias sociales, y la Ley Orgánica 3/2018, de 5 de diciembre, de Protección de Datos Personales y garantía de los derechos digitales (2018).

\section{Análisis de datos}

El análisis de los datos se realizó con la ayuda del paquete estadístico SPSS, versión 25 . Se ha recurrido a la estadística descriptiva. Dentro de ella se ha utilizado la distribución de frecuencias y porcentajes, medidas de tendencia central (media), medidas de variabilidad (desviación estándar) y representación en gráficos de distintas tipologías. Además, se aplicó la prueba de bondad de ajuste Shapiro Wilks y la Chi-cuadrado de Pearson para comprobar la asociación o relación entre variables.

\section{Resultados}

Los resultados se estructuran en torno a los objetivos propuestos con anterioridad y a dos de las dimensiones que conforman el instrumento de diagnóstico socioeducativo aplicado y que se analizan a continuación.

No obstante, en primer lugar, para contrastar la normalidad de los datos, se aplicó el Test de Shapiro Wilks. Los resultados de la ejecución de dicha prueba arrojaron p-valores inferiores a 0.05 en todas las variables, por lo que se puede afirmar que el comportamiento de las mismas es no normal.

En segundo lugar, y puesto que las variables de estudio no se ajustan a la distribución normal, se optó por aplicar la Chi-cuadrado de Pearson para analizar la posible relación entre las variables sexo (dicotómica), etapa educativa (politómica) y experiencia de los docentes (dicotómica) respecto a las dificultades de los maestros y maestras en sus funciones docentes (tipo Likert con cinco (5) opciones de respuesta) y las posibles soluciones para hacer frente a dichos obstáculos (tipo Likert con cinco (5) opciones de respuesta). Los p-valores arrojados por dicha prueba, mayores a 0.05 , pusieron de manifiesto la no existencia de relación o asociación entre variables. Por este motivo, el análisis que se llevó a cabo fue meramente descriptivo.

De esta forma, en relación con el objetivo $a$ (describir las dificultades en el centro educativo y en el aula señaladas por el profesorado en general), los principales problemas a los que los docentes encuestados deben hacer frente en su labor diaria son, por orden de valoración, los siguientes: la sobrecarga de trabajo, la motivación de los alumnos, el mantenimiento de la disciplina, el trabajo burocrático, el número de alumnos por aula, las relaciones con los alumnos, la adaptación de la enseñanza a las diferencias individuales, los problemas con alumnos en concreto y el tratamiento de los alumnos de aprendizaje lento, así como la falta de tiempo para preparar las clases. En la tabla 2 quedan recogidas las medias y desviaciones típicas de todos los ítems de la dimensión 1 . 
Tabla 2. Medias y desviaciones típicas de los ítems que conforman la variable problemas en el desempeño de las funciones docentes

\begin{tabular}{|c|c|c|}
\hline & Media & $\begin{array}{c}\text { Desviación } \\
\text { típica }\end{array}$ \\
\hline La disciplina en el aula & 4.13 & 1.167 \\
\hline La motivación de los alumnos & 4.30 & 1.022 \\
\hline Adaptación de la enseñanza a las diferencias individuales & 3.77 & 1.006 \\
\hline Evaluar a los alumnos & 3.30 & .915 \\
\hline Las relaciones con los padres & 3.27 & 1.112 \\
\hline La organización del trabajo en clase & 3.24 & 1.380 \\
\hline Insuficiencia de material & 3.30 & 1.208 \\
\hline Problemas con alumnos en concreto & 3.72 & 1.099 \\
\hline Sobrecarga de trabajo & 4.33 & .922 \\
\hline Relaciones con los otros profesores & 2.55 & 1.404 \\
\hline Falta de tiempo para preparar las clases (la programación diaria) & 3.57 & 1.305 \\
\hline El dominio de los diferentes métodos de enseñanza & 3.07 & 1.172 \\
\hline Vigilancia de las normas de la escuela & 3.00 & 1.174 \\
\hline Determinar el nivel de aprendizaje de los alumnos & 2.79 & 1.292 \\
\hline Dominio de las materias & 3.03 & 1.497 \\
\hline El trabajo burocrático & 4.07 & 1.143 \\
\hline Relaciones con el Equipo Directivo & 2.60 & 1.192 \\
\hline Materiales didácticos inadecuados & 3.10 & 1.125 \\
\hline Tratamiento de alumnos de aprendizaje lento & 3.57 & 1.104 \\
\hline Tratamiento de la interculturalidad & 3.00 & 1.259 \\
\hline Dominio de los libros de texto y guías curriculares & 2.50 & 1.253 \\
\hline Falta de tiempo libre & 3.07 & 1.172 \\
\hline Falta de apoyo y orientación & 3.33 & 1.241 \\
\hline El número de alumnos por aula & 3.87 & 1.358 \\
\hline Las relaciones con los alumnos & 3.78 & 1.340 \\
\hline
\end{tabular}

Fuente: elaboración propia. Los ítems analizados en esta tabla pertenecen al instrumento de Cardona (2008)

El objetivo $b$ pretende especificar las soluciones más importantes para mejorar el desempeño de las funciones docentes, distinguiendo entre las que están dirigidas al grupo en general, a los profesores principiantes y a los experimentados.
Así pues, las medidas detectadas para el grupo de docentes noveles se representan gráficamente (véase figura 3). 


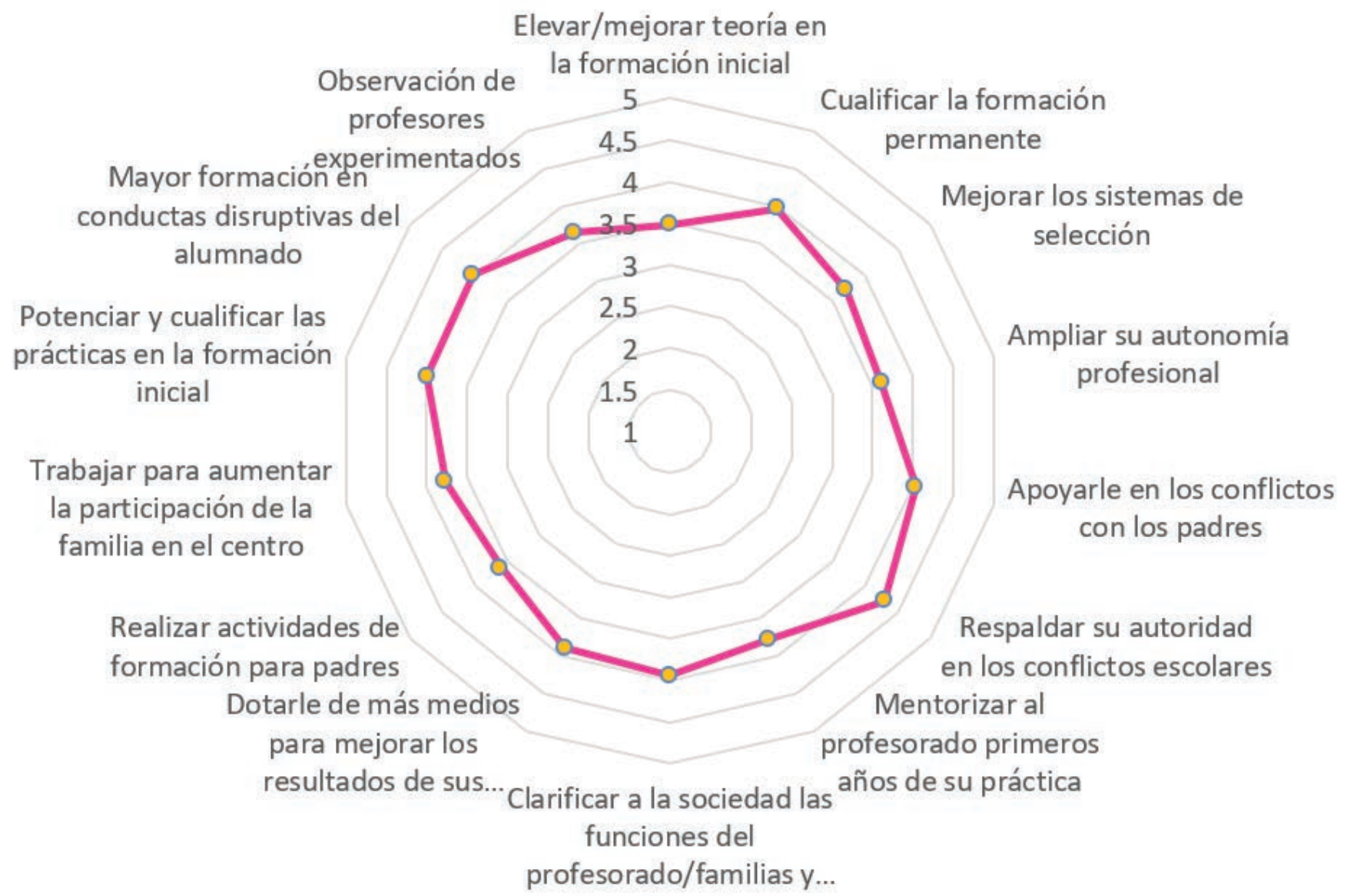

Figura 3. Medidas para evitar o disminuir las dificultades en las funciones docentes del profesorado principiante.

Fuente: elaboración propia. Los ítems analizados en esta figura pertenecen al instrumento de Cardona (2008).

Como se aprecia en la figura 3, los profesores y las profesoras encuestadas consideran que las principales medidas para evitar las dificultades en las funciones docentes del profesorado principiante son: a) respaldar su autoridad en los conflictos escolares en general $(\mathrm{M}=4.29$; $\mathrm{DT}=.58), \mathrm{b})$ más formación en el conocimiento de las principales conductas disruptivas del alumnado $(M=4.03$; DT=.75), c) apoyarles en los conflictos con los progenitores $(4.03$; DT $=.70) \mathrm{y}$ d) potenciar y cualificar las prácticas en la formación inicial $(\mathrm{M}=4$; $\mathrm{DT}=.91)$. Dos de las medidas más señaladas apuntan a cambios en la formación inicial y permanente de los profesores y profesoras en activo y también de los futuros egresados universitarios. Las otras dos soluciones apuntadas se relacionan con el soporte y refuerzo que requieren maestros y maestras del equipo docente de sus centros educativos, del equipo directivo y de la sociedad en general cuando se dan problemas de convivencia en el ámbito escolar y con miembros con los que se relacionan desde estas inmediaciones, como son los padres y las madres.

Finalmente, las soluciones para paliar los problemas en la función docente del profesorado experto y las seleccionadas para dar respuesta a las dificultades del profesorado en general, quedan recogidas en la tabla 3 y en la tabla 4, respectivamente. 
Respecto a las medidas para solventar o paliar las dificultades en las funciones docentes en el profesorado experimentado, los maestros y maestras encuestadas consideran que es prioritario respaldar su autoridad en los conflictos escolares ( $\mathrm{M}=4.47$; DT $=.68)$, apoyarlos en los conflictos con los progenitores $(\mathrm{M}=4.37$; DT =.71), incentivar el intercambio de experiencias y buenas prácticas $(\mathrm{M}=4.33 ; \mathrm{DT}=.75)$ y clarificar a la sociedad las funciones del profesorado, de las familias y las que deben ser objeto de actuación conjunta $(\mathrm{M}=4.27$; DT=.69).

Tabla 3. Medias y desviaciones típicas de los ítems que conforman la variable medida para mitigar los obstáculos a los que se enfrentan los docentes experimentados

\begin{tabular}{|l|c|c|}
\hline & Media & $\begin{array}{c}\text { Desviación } \\
\text { típica }\end{array}$ \\
\hline Elevar y mejorar la teoría en la formación inicial & 3.50 & 1.137 \\
\hline Mejorar la formación permanente & 4.27 & .907 \\
\hline Mejorar los sistemas de selección & 3.80 & 1.095 \\
\hline Ampliar su autonomía profesional & 3.93 & 1.048 \\
\hline Apoyarle en los conflictos con los padres & 4.37 & .718 \\
\hline Respaldar su autoridad en los conflictos escolares & 4.47 & .681 \\
\hline $\begin{array}{l}\text { Clarificar a la sociedad las funciones del } \\
\text { profesorado, de las familias y las que deben ser } \\
\text { objeto de actuación conjunta }\end{array}$ & 4.27 & .691 \\
\hline $\begin{array}{l}\text { Dotarle de más medios para mejorar los resultados } \\
\text { de sus alumnos }\end{array}$ & 4.23 & .774 \\
\hline $\begin{array}{l}\text { Trabajar para aumentar la participación de la } \\
\text { familia en el centro }\end{array}$ & 3.93 & .944 \\
\hline $\begin{array}{l}\text { Potenciar y cualificar las prácticas en la formación } \\
\text { inicial }\end{array}$ & 3.72 & .996 \\
\hline $\begin{array}{l}\text { Mayor formación en el conocimiento de las } \\
\text { principales conductas disruptivas del alumnado }\end{array}$ & 4.17 & .747 \\
\hline $\begin{array}{l}\text { Incentivar el intercambio de experiencias y buenas } \\
\text { prácticas }\end{array}$ & 4.33 & .758 \\
\hline
\end{tabular}

Fuente: elaboración propia. Los ítems analizados en esta tabla pertenecen al instrumento de Cardona (2008).
En cuanto a las medidas para paliar o solventar los problemas en las funciones docentes en el profesorado en general, los profesionales encuestados consideran que es muy necesario potenciar la valoración de lo actitudinal y lo emocional en el alumnado $(\mathrm{M}=4.27$; DT =.86), así como profundizar en la calidad humana de las relaciones profesor-familia-alumno/a $(\mathrm{M}=4.27$; $\mathrm{DT}=.82)$. El resto de ítems pueden consultarse en la tabla 4.

Tabla 4. Medias y desviaciones típicas de los ítems que conforman la variable medida para mitigar los obstáculos a los que se enfrentan los docentes en general

\begin{tabular}{|l|c|c|}
\hline & Media & $\begin{array}{c}\text { Desviación } \\
\text { típica }\end{array}$ \\
\hline $\begin{array}{l}\text { Facilitar una mayor formación del } \\
\text { profesorado en metodología didáctica }\end{array}$ & 4.03 & .809 \\
\hline $\begin{array}{l}\text { Potenciar la valoración de lo } \\
\text { actitudinal y lo emocional en el } \\
\text { alumnado }\end{array}$ & 4.27 & .868 \\
\hline $\begin{array}{l}\text { Impulsar el desarrollo de estrategias } \\
\text { de enseñanza-aprendizaje }\end{array}$ & 4.20 & .847 \\
\hline $\begin{array}{l}\text { Sistematizar la reflexión en y sobre la } \\
\text { práctica }\end{array}$ & 4.13 & .900 \\
\hline $\begin{array}{l}\text { Profundizar en la calidad humana de } \\
\text { las relaciones profesor-familia-alumno }\end{array}$ & 4.27 & .828 \\
\hline $\begin{array}{l}\text { Buscar un equilibrio armónico entre } \\
\text { la función gestora y educativa en el } \\
\text { centro y aula }\end{array}$ & 4.03 & .890 \\
\hline $\begin{array}{l}\text { Incrementar el trabajo en equipo de } \\
\text { los docentes }\end{array}$ & 4.20 & .805 \\
\hline $\begin{array}{l}\text { Ampliar la presencia de educadores y } \\
\text { trabajadores sociales en los centros } \\
\text { humanos, curriculares, culturales y } \\
\text { materiales del entorno }\end{array}$ & 4.07 & .828 \\
\hline $\begin{array}{l}\text { Dotar de mayores recursos a los } \\
\text { Oervicios de Orientación Educativa }\end{array}$ & 4.17 & .834 \\
\hline
\end{tabular}

Fuente: elaboración propia. Los ítems analizados en esta tabla pertenecen al instrumento de Cardona (2008). 


\section{Conclusiones}

Desde el análisis realizado sobre los datos se observó que los docentes registran la sobrecarga de trabajo en el primer lugar de importancia de las dificultades a las que se enfrentan en su profesión. A partir de las condiciones imperantes en la posmodernidad se generan nuevas tareas en el quehacer docente (Martín-Romera y García-Martínez, 2018; Tejada y Pozos, 2018), entre las que se destacan, según Ávalos, Cavada, Pardo, y Sotomayor, (2010), las que siguen:

Reuniones entre colegas, preparación de proyectos, tutorías a compañeros de trabajo, ejercicios de inducción a nuevos colegas, participación en actividades de desarrollo colectivas, integración de comisión para analizar los casos difíciles de los estudiantes, reuniones e informaciones escritas a los padres y, en general, aumento del trabajo burocrático (p. 243).

Todo ello constituye un peso que se suma a las responsabilidades que demanda del docente la sociedad y que es causante de su sobrecarga laboral. Ahora bien, la sobrecarga laboral se constata como una de las dificultades que han sido reiteradas en orden de citación por el profesorado tanto en estudios recientes y no tan recientes y a ello se ha asociado otras preocupaciones que igualmente persisten tales como el elevado número de estudiantes en el aula, el trato diferencial de estudiantes. Esto se constata en los estudios de Eirín, García y Montero (2009b), Moliner y Ortí (2016), Montero y Gevere, (2018).

De otro lado, se registra la motivación de los alumnos como la segunda de las dificultades enunciadas por los docentes en orden de importancia, y es interesante que otros estudios realizados recientemente, como por ejemplo el de Moliner y Ortí (2016), verifiquen el tema de la motivación de los alumnos y alumnas como un asunto considerado problemático para los docentes.

Esto es comprensible, puesto que desde los trabajos de Weiner, con su teoría atribucional, la teoría de las metas de aprendizaje de Dweck y la teoría de Nicholls, los constructos motivacionales, afectivos, además de los cognitivos, constituyen algunas de las variables que varios autores enfocan en relación con los procesos de enseñanza y aprendizaje (García y Domenech, 2005), siendo las perspectivas socio-constructivistas, con exponentes como Bandura (1991), Pintrich y Shunk (2006) y Zimmerman y Shunk (2008), entre otros, los más destacados.

Asimismo, las variables personales van a determinar en grado sumo la motivación escolar y, tal como señalan Maquilón y Hernández (2011), "son referencia obligada de todo profesor que desee incidir en la motivación de sus estudiantes" (p. 86). Por tanto, que este aspecto haya sido privilegiado por los docentes de estudio refleja su pertinencia dentro de los desafíos escolares actuales.

El manejo de la disciplina apareció en el tercer orden de acuerdo a la valoración concedida por los docentes que conformaron la muestra de estudio. Este hecho deja ver que es una de las dificultades más apremiantes a las que se enfrentan estos docentes (Moliner y Ortí, 2016). No extraña tal resultado y coincide con las reflexiones aportadas por autores como Alfaro (2011), quien destaca las modificaciones en la relación entre docente-estudiante, ya que, dada la influencia de los profundos cambios de la modernidad sobre el sistema de cosas actuales, pasó de ser vertical y unidireccional (en donde el docente custodiaba la verdad, era dueño de la información y el alumno se sometía a la autoridad y disciplina sin cuestionar a su maestro) a horizontal y bidireccional. Hoy, en contraste, "el profesorado no tiene asegurada la escucha, el respeto y la admiración de sus estudiantes" (Sánchez-Sánchez y Jara-Amigo, 2019, p. 3). Esto muy probablemente contribuye a que el manejo de la disciplina sea una de las dificultades de mayor preocupación para el grupo de profesores y profesoras del estudio.

En cuarto orden de valoración, los docentes apuntaron al trabajo burocrático como otra de las dificultades que deben lidiar. Al respecto, señalaba Weber (2007) que, debido al ambiente de reformas regidas por los principios de una economía neoliberal, un ambiente turbulento de políticas, el resultado es la consecuente intensificación de tareas que a partir de estas tensiones debe realizar el profesorado (Van der Berg, 2002; Ávalos et al., 2010). En este sentido, coincide de forma interesante con los trabajos de 
Cardona (2008) y, posteriormente, con los de Moliner y Ortí (2016), los cuales apuntan al trabajo burocrático como una de las problemáticas de mayor protagonismo entre el profesorado.

Así pues, este aumento de los modos burocráticos manifestado por los contrastes y conflictos entre políticas y su implementación, y entre estas y las demandas de adecuar la enseńanza a las individualidades y estilos de aprendizaje, constituyen sin duda, como lo han reafirmado estos docentes, un verdadero óbice en la labor del profesorado actual. Por otro lado, se sugiere una relación entre este aumento del trabajo burocrático y la sobrecarga laboral, puesto que, como se revisó anteriormente, la burocracia implica y demanda nuevas tareas.

En lugares sucesivos se encuentran el número de alumnado por aula, las relaciones con los estudiantes, las adaptaciones, significativas o no, que el docente debe llevar a cabo para atender a las diferencias individuales, los problemas con niños, niñas o adolescentes en particular y el tratamiento de los alumnos y alumnas con aprendizaje lento (no teniéndose en cuenta aquellos que llevan un aprendizaje más rápido que el resto). Todas estas dificultades, como se observa, recaen directa o indirectamente sobre las relaciones entre docente-estudiante.

En el caso del tamaño de la clase, o la proporción docente-alumno, además de ser uno de los obstáculos enunciados por los docentes, es uno de los problemas que inciden en una educación de calidad (Organización de las Naciones Unidas para la Educación, la Ciencia y la Cultura, UNESCO, 2015, 2016). Así lo destacan diferentes estudios al señalar que el número de alumnos y alumnas impacta, por ejemplo, en el clima de aula, presentando un efecto negativo en relación con el rendimiento y desempeño académico. Esto parece deberse a la dispersión que se produce en el salón de clase, lo cual repercute a su vez en la tergiversación de los esfuerzos de los maestros y maestras (Monks y Smith, 2011; Moliner y Ortí, 2016; Rodríguez y Tascón, 2017). De ahí que sea plausible suponer el motivo de preocupación para estos profesores y profesoras.

De otro lado se destacan las adaptaciones que, diseñadas por el docente o por otros profesionales del escenario educativo, se deben poner en marcha en el proceso de enseñanza-aprendizaje para dar respuesta a las necesidades de los alumnos y alumnas, las cuales pueden ser muy diferentes. Al respecto, es comprensible porque los docentes apuntaron a estos aspectos, pues como se ha señalado anteriormente, el desarrollo de la sociedad actual ha dejado patente la heterogeneidad, disparidad y diversidad en las situaciones, contextos y también en las personas (Pérez, Martínez, Tey, Essombra y González, 2007) y es esencial el saber conocer a los alumnos y alumnas (Espino et al., 2019). Por consiguiente, la escuela decimonónica tradicional y su afán por transmitir de forma hegemónica los contenidos, cerrarse a una única metodología de enseñanza y tratar a todos los estudiantes de la misma forma han caducado, quedando obsoletos (Ávalos et al., 2010; March y Orte, 2014). De tal forma que los docentes están presionados a satisfacer el derecho a aprender de sus estudiantes, respetando sus diferencias e individualidades, entre las cuales se incluyen la diversidad cultural, lingüística y étnica, muy común en la población estudiantil variopinta de las aulas españolas debido a las olas de inmigración.

Asimismo, se suma a ello el creciente interés por los estilos de aprendizaje, desde los trabajos de Kolb (1976), Vermunt (1996), Boekaerts (1999), entre otros autores, $y$, en consecuencia, la preocupación del docente por el uso eficaz y eficiente de estos estilos, que dada su naturaleza compleja, por cuanto se verifican interacciones entre las exigencias del entorno o el medio social con las características personales del estudiante, resulta ser un proceso en el que intervienen múltiples variables o constructos de carácter afecto-motivacional-cognitivo (Aguilera y Ortiz, 2009; Olivo, 2017; Olivo, 2019; Sánchez y Andrade, 2014).

Así pues, las dificultades a las que tiene que hacer frente el profesorado actual en el ejercicio de su profesión docente no pueden describirse sin tener en cuenta los estudios anteriores, como el de Veenman (1984) y Cardona (2008). Como se observa en la figura 4, existen aspectos que se repiten y son una constante a lo largo del tiempo, como también lo señalan Moliner y Ortí (2016), tales como el mantenimiento de la disciplina en el aula, la sobrecarga laboral por el trabajo burocrático, el número de alumnos, y otros que desaparecen y afloran. 


\section{Año 1984}

- Falta de tiempo para preparar las clases

- Número de alumnos por aula

- Insuficiencia de materiales

- Falta de tiempo libre

- Escasez de instalaciones en el centro

- Disciplina en el aula
Año 2008

- Trabajo burocrático

- Motivación del alumnado

- Adaptación de la enseñanza a las diferencias individuales

- Disciplina en el aula

- Falta de tiempo para preparar las clases

- Falta de tiempo libre

\section{Actualidad}

- Sobrecarga de trabajo

- Motivación del alumnado

- Disciplina en el aula

- Trabajo burocrático

- Número de alumnos por aula

- Relaciones con los alumnos y familia

- Adaptación de la enseñanza a las diferencias individuales

Figura 4. Principales problemas del profesorado en su función docente. Comparativa de estudios.

Fuente: elaboración propia

Nota: los resultados de los estudios expuestos en la figura 4 son los pertenecientes a Veenman (1984), Cardona (2008) y el presentado en este texto.

Por otra parte, con respecto a las medidas para hacer frente a los problemas en las funciones docentes en el profesorado en general, es de interés resaltar que los profesionales encuestados privilegiaron potenciar la valoración de lo actitudinal y lo emocional en el estudiantado, así como profundizar en la calidad humana de las relaciones profesor-familia-alumno/a.

Esto puede denotar la conciencia de tales docentes por las tendientes corrientes socioconstructivistas y teorías psicoeducativas, las cuales conceden protagonismo a aspectos de dimensión socioafectiva y emocional de los estudiantes para promover aprendizajes exitosos (Olivo, 2017). También a la imperiosa necesidad de poner en marcha programas formativos para padres y madres desde los centros educativos para el desarrollo de los principios para una parentalidad positiva (Martínez, Rodríguez, Álvarez y Becedóniz, 2016; Rubio, 2019), puesto que podrían favorecer las relaciones de profesores y profesoras con sus estudiantes y también con el resto de los miembros de las familias (Rodrigo, Martínez y Rodríguez, 2018).

Así pues, existe una diferencia en comparación con lo indicado por Cardona (2008), y es que los docentes encuestados sí consideran como relevante un mayor apoyo en los conflictos con los padres y madres de sus discentes.

De igual forma, es preciso señalar que el presente estudio no está exento de limitaciones, las cuales es preciso orientarlas a prospectivas en investigaciones futuras.

En primer lugar, la muestra es pequeña y seleccionada de forma no aleatoria, por lo que no es posible generalizar los resultados.

En segundo lugar, los informantes han sido mayoritariamente mujeres y pertenecientes a los cuerpos de docentes de educación infantil, primaria y secundaria, no habiéndose explorado las voces del profesorado de educación superior, formación profesional y educación no formal. Además, la mayor parte de los participantes fueron profesoras y profesores experimentados.

En tercer lugar, los resultados se basan en la aplicación de un instrumento cuantitativo de autoinforme, por lo que sería conveniente llevar a cabo, por ejemplo, grupos de discusión con docentes para conocer sus dificultades desde un enfoque cualitativo y que permitiese profundizar en dichas problemáticas y en otras no diagnosticadas hasta el momento. 
Ahora bien, sería interesante abordar las dificultades del profesorado relacionadas particularmente con la tendiente diversidad familiar, por ejemplo, porque aunque en este trabajo no se dio cuenta de ello, sin duda suponen un desafío adicional a la ya compleja labor de los docentes de hoy.

Para finalizar, un aspecto que no ha salido a la luz en este estudio, y sin duda es pertinente, es el desafío que supone en la profesión docente la inminente presencia de una sociedad tecnológica. Este contexto rodea a los centros educativos, configurando nuevas situaciones que en un sinnúmero de ocasiones constituyen un repertorio de posibilidades-limitaciones. De tal forma, la complejidad del factor tecnológico implica en algunos casos, como apuntan Sánchez-Sánchez y Jara-Amigo (2019), "un limitante (inhibidora) del trabajo docente y de la educación misma, en tanto lo dificulta y lo entorpece” (p. 15). Vale la pena revisar de cerca la tensión provocada por los entornos virtuales de aprendizaje, pertenecientes a las tecnologías de la información y la comunicación, en la lógica tradicional y en el entendimiento con el que se ha concebido la tarea docente.

\section{Referencias}

Abril, A. y Peinado, M. (2018). Nuevos horizontes familiares: Una reflexión en la formación del profesorado. Profesorado. Revista de curriculum y formación del profesorado, 22(1), 159-176. Recuperado de https://recyt.fecyt.es/index.php/ profesorado/article/view/63639/38697

Aguilera, E; y Ortiz, E. (2009). Las investigaciones sobre los estilos de aprendizaje sus modelos explicativos. Revistas Estilos de aprendizaje, 4(4), 1-19. Recuperado en https://cutt.ly/grF1qhT

Alfaro, T. (2011). Desafío docente: el alumno postmoderno. Revista digital de Investigación en Docencia universitaria, 5(1), 54-65. Doi: https://doi.org/ $10.19083 /$ ridu. 5.4

Álvarez, C. (2015). Teoría frente a práctica educativa: algunos problemas y propuestas de solución. Perfiles Educativos, 37(148), 172-190. Doi: https://doi.org/10.22201/iisue.24486167e. 2015.148.49320
Ávalos, B., Cavada, P., Pardo, M. y Sotomayor, C. (2010). La profesión docente: temas y discusiones en la literatura internacional. Estudios Pedagógicos, 34(1), 253-263. Doi: https://doi. org/10.4067/s0718-07052010000100013

Bandura, A. (1991). Social Cognitive Theory of self-regulation. Organizational Behavior and Human Decision Process, 50, 248-287. Recuperado de http://www.uky.edu/ - eushe2/BanduraPubs/ Bandura1991OBHDP.pdf

Boekaerts, M. (1999). Motivated learning: Studying students situation transactional unit. European Journal of Pychology of Education, 14(1), 41-55. Recuperado de https://cutt.ly/XrFM4LG

Cardona, J. (2008). Problemática actual del profesorado. Algunas soluciones. Enseñanza, 26, 29-56. Recuperado de https://cutt.ly/YrFxTcl

Cardona, J. (2008b). Formación y Desarrollo Profesional del Docente en la Sociedad del Conocimiento. Madrid: Universitas.

Cardona, J. (2013). Cuestiones en torno a la Formación y Desarrollo Profesional de los Profesores. Madrid: Sanz y Torres.

Cerezo, F. y Rubio, F.J. (2017). Medidas relativas al acoso escolar y ciberacoso en la normativa autonómica espańola. Un estudio comparativo. Revista Electrónica Interuniversitaria de Formación del Profesorado (REIFOP), 20(1), 113-126. Doi: https://doi.org/10.6018/reifop/ 20.1.253391

Day, Ch. (2005). Formar docentes. Cómo, cuándo y en qué condiciones aprende el profesorado. Madrid: Narcea.

Eirín, R., García, M. y Montero, L. (2009a). Profesores principiantes e iniciación a la docencia. Estudio exploratorio, Revista de currículum y formación del profesorado, 13(1), 1-15. Recuperado de https:// www.ugr.es/ -recfpro/rev131ART7.pdf

Eirín, R., García, M. y Montero, L. (2009b). Desarrollo profesional y profesionalización docente. Perspectivas y problemas. Profesorado, Revista de curriculum y formación del profesorado, 13(2), 1-13. Recuperado de https://recyt.fecyt.es/index. php/profesorado/article/view/42344 
Espino, H., Galván, L., y Blanco, N. (2019). Saber conocer a los alumnos en el umbral de la profesión docente. CPU-e. Revista de Investigación Educativa, 29, 108-130. Recuperado de http://cpue. uv.mx/index.php/cpue/article/view/2635/4501

Flores, A. (2015). Dificultades laborales de profesores en escuelas secundarias. Educación y Educadores, 18(3), 411-431. Doi: http://dx.doi.org/10.5294/ edu.2015.18.3.3

Franco, J., López, H. y Arango, D. (2020). La satisfacción de ser docente. Revista Complutense de Educación, 31(1), 55-67. Doi: http://dx.doi. org/10.5209/rced.61775

García, M. y Domenech, D. (2005). Motivación, aprendizaje y rendimiento escolar. REME. Revista electrónica de motivación y emoción, 1, 55-66. Recuperado de: http://reme.uji.es/articulos/ pa0001/texto.html

García, M.P. y Martínez, P. (coords.). (2012). Guía práctica para la realización de Trabajos de Fin de Grado y Trabajos de Fin de Máster. Murcia: Ediciones de la Universidad de Murcia.

Hargreaves, A. (2005). Profesorado, cultura y postmodernidad: cambian los tiempos, cambia el profesorado, Madrid: Morata.

Hernández, R. y Mendoza, C.P. (2018). Metodología de la investigación. Las rutas cuantitativa, cualitativa $y$ mixta. Ciudad de México: McGraw-Hill.

Imbernón, F. (1998). La formación y el desarrollo profesional del profesorado. Hacia una nueva cultura profesional. Barcelona: Graó.

Jiménez, A., Jara, M. y Miranda, E. (2012). Burnout, apoyo social y satisfacción laboral en docentes. Psicología Escolar Educacional, 16(1), 125-134. Recuperado de http://www.scielo.br/pdf/pee/ v16n1/13.pdf

Kolb, D. (1976). On management and the learning process. California Management Review, 18(3), 21-21. Recuperado de https://cutt.ly/trFM28c

Ley Orgánica 3/2018, de 5 de diciembre, de Protección de Datos Personales y garantía de los derechos digitales (2018). Boletín Oficial del Estado (BOE), 294, de 6 de diciembre, 9788- 119857.

Maquilón, J. y Hernández, F. (2011). Influencia de la motivación en el rendimiento académico de los estudiantes de formación profesional. REIFOP, 14(1), 81-100. Recuperado de https://cutt.ly/ MrFMYKk

Marcelo, C. (1991). Dimensiones ambientales en clase de profesores principiantes según el C.U.C.E.I., Enseñanza, 8, 19-33. Recuperado de: https://dialnet.unirioja.es/servlet/articulo? codigo $=4551045$

Marcelo, C. (1999). Estudio sobre estrategias de inserción profesional en Europa, Revista Iberoamericana de Educación, 19, 101-144. Recuperado de http://rieoei.org/oeivirt/rie19a03.htm

Marcelo, C. (coord.) (2008). El profesorado principiante. Inserción a la docencia. Barcelona: Octaedro.

March, M.X. y Orte, C. (2014). La educación social en la sociedad del siglo XXI. En M. Marti y $C$. Orte (Coord.), La pedagogía socialy la escuela. Los retos socieducativos de la institución escolar del siglo XXI (pp. 19-56). Barcelona: Octaedro.

Martínez, R.A., Rodríguez, B., Álvarez, L. y Becedóniz, C. (2016). Evidence in promoting positive parenting through the Program-Guide to Develop Emotional Competences. Psychosocial Intervention, 25(2), 111-117. Doi: https://doi. org/10.1016/j.psi.2016.04.001

Martín, A. y García, I. (2018). Profesionalización del docente en la actualidad. Profesorado. Revista de curriculum y formación del profesorado, 22(1), 7-23. Recuperado de: https://dialnet.unirioja. es/servlet/articulo?codigo $=6343806$

Molas, N. y Rosselló, M. (2010). La introducción de las TIC en las aulas y el nuevo rol docente [en línea]. Recuperado de http://www.raco.cat/index.php/ $\mathrm{dim} /$ article/viewFile/214711/285003

Moliner, L. y Ortí, J. (2016). ¿Podré acabar el temario?: Las preocupaciones a las que se enfrenta el profesorado novel. Revista Complutense de Educación, 27(2), 827-844. Doi: http://dx.doi. org/10.5209/rev_RCED.2016.v27.n2.48371

Monks, J. y Smith, R.M. (2011). The impact of class size on outcomes in higher education. The $B E$ Journal of Economic Analysis \& Policy, 11(1), 1-12. Doi: https://doi.org/10.2202/19351682.2803 
Montero, L. (1999). Formación y desarrollo profesional: cruce de miradas. XXI, Revista de Educación, 1, 15-31. Recuperado de https://dialnet. unirioja.es/servlet/articulo? codigo $=201090$

Montero, L. y Gewerc, A. (2018). La profesión docente en la sociedad del conocimiento. Una mirada a través de la revisión de investigaciones de los últimos 10 años. RED. Revista de Educación a Distancia, 56(3), 1-22. Doi: http://dx.doi. org/10.6018/red/56/3

Olivo, J. (2017). Caracterización de estudiantes exitosos: Una aproximación al aprendizaje de Ciencias Naturales. CPU-e Revista de Investigación Educativa, 25, 114-143. Doi: https://doi. org/10.25009/cpue.v0i25.2528

Olivo, J. (2019). Interpretativa de docentes de Ciencias Naturales sobre estudiantes exitosos, Revista Complutense de Educación 30(2), 17-34. Doi: https://doi.org/10.5209/RCED. 57395

Organización de las Naciones Unidas para la Educación, la Ciencia y la Cultura [UNESCO] (2015). Informe de Seguimiento de la EPT en el mundo. Recuperado de https://cutt.ly/nrFMSjd

Organización de las Naciones Unidas para la Educación, la Ciencia y la Cultura [UNESCO] (2016). Educación 2030. Declaración de Inchon y Marco de Acción Hacia una educación inclusiva y equitativa de calidad y un aprendizaje a lo largo de la vida para todos. Recuperado de https://cutt. ly/IrFMPB7

Pérez, A., Martínez, E., Tey, A. Essombra, M y González, T. (2007). Profesorado y otros profesionales de la Educación. Madrid: Octaedro.

Perrenoud, P. (2011). Desarrollar la práctica reflexiva en el oficio de enseñar. Barcelona: Graó.

Pintrich, P. R. y Shunk, D. H. (2006). Motivación en contextos educativos. Teoría, investigación y aplicaciones. Madrid: Pearson.

Rodrigo, M.J., Martínez, R.A. y Rodríguez, B. (2018). La relación centro escolar-familia como factor protector de conductas transgresoras en la adolescencia. Aula abierta, 47(2), 149-158. Doi: https://doi.org/10.17811/rifie. 47.2.2018.149-158

Rodríguez, M. y Tascón, D. (2017). Impacto del número de estudiantes en un mismo salón sobre los resul- tados de las pruebas de estado para Colombia. Vniversitas Económica, 17(2), 1-33. Recuperado de: https://cutt.ly/prFMZBh

Roig, R., Mengual, S. y Quinto, P. (2014). Conocimientos tecnológicos, pedagógicos y disciplinares del profesorado de Primaria. Comunicar, 23(45), 151-159.

Ros, A. y Chisvet, M. (2018). Las investigaciones sobre las teorías implícitas del profesorado de formación profesional en el estado español. Profesorado. Revista de curriculum y formación del profesorado, 22(1), 97-115. Recuperado de https://recyt.fecyt.es/index.php/profesorado/ article/view/63623/38694

Rubio, F. J. (2019). Programas promotores de parentalidad positiva. Protocolo para una revisión sistemática. En Belmonte, Gázquez, Simón, Soriano, Oropesa, Martos y Barragán (Eds.), Innovación Docente e Investigación en Ciencias Sociales, Económicas, y Jurídicas. Madrid, España: editorial Dykinson. Recuperado de: https:// cutt.ly/drF0aBW

Rubio, F. J., Díaz, A. y Cerezo, F. (2019). Bullying y cyberbullying: la respuesta de las comunidades taria de Formación del Profesorado (REIFOP), 25(1), 145-157. Doi: https://doi.org/10.6018/ reifop.22.1.332311

Sánchez, A., Boix, J. L. y Jurado, P. (2009). La sociedad del conocimiento y las TICs: una inmejorable oportunidad para el cambio docente. Pixel-Bit, Revista de Medios y Educación, 34, 179-204. Recuperado de: https://www.redalyc.org/pdf/ 368/36812036013.pdf

Sánchez, L. y Andrade, R. (2014). Inteligencias múltiples $y$ estilos de aprendizaje. México: Alfaomega.

Sánchez, M.C. (2007). El estrés docente, un grave problema para la enseñanza. [Texto en línea]. Recuperado de http://www.educaweb.com/ noticia/2007/01/15/estres-docente-grave-problema-ensenanza-2146/

Sánchez, G. y Jara, X. (2019). Estudiantes, docentes y contexto educativo en la representación del profesorado en formación. Revista electrónica Educare, 23(3), 1-21. Doi: https://doi. org/10.15359/ree.23-3.8 
Tejada, J. y Pozos, K. (2018). Nuevos escenarios y competencias digitales docentes: Hacia la profesionalización docente con TIC. Profesorado. Revista curriculum y formación del profesorado, 22(1), 25-51. Recuperado de https:// recyt.fecyt.es/index.php/profesorado/article/ view/63620

Tovar, J. (2018). Formación investigativa de profesores: El problema como producto del proceso de problematización. Profesorado. Revista curriculum y formación del profesorado, 22(1), 117-132. Recuperado de https://recyt.fecyt.es/index.php/ profesorado/article/view/63625/38695

Van Dalen, D.B. (1990). Manual de técnica de investigación educacional. México: Paidós.

Van der Berg, R. (2002). Teachers meanings regarding educational practice. Review of educational Research, 72(4), 577-565. Doi: https:// doi.org/10.3102/00346543072004577

Vázquez, E. (2016). Dificultades del profesorado para planificar, coordinar y evaluar competencias claves. Un análisis desde la inspección de educación. Revista Complutense de Educación, 27(3), 1062-1083. Doi: http://dx.doi.org/10.5209/ rev_RCED.2016.v27.n3.47400

Veenman, S. (1984). Perceived Problems of Beginning Teachers. Review of Educational Research, 54(2), 143-178. Doi: https://doi.org/10.2307/1170301
Veeman, S. (1988). El proceso de llegar a ser: un análisis de la formación inicial. En Villa, A (coord.). Perspectivas y problemas de la función docente (pp. 39-68). Madrid: Narcea.

Vermunt, J.D. (1996). Metacognitive, cognitive and affective aspects of learning styles and strategies: A phenomenographic analysis. Higher Education, 31(1), 25-50. Doi: https:/doi.org/ $10.1007 / \mathrm{bf00129106}$

Weber, E. (2007). Globalization, "Glocal” Deveploment teachersWork: A research agenda. Review of Educational Research, 77(3), 279-309. Doi: https://doi.org/10.3102/003465430303946

Zayas, B., Gonzálvez, V. y García, J. (2019). La dimensión ética y ciudadanía del aprendizaje servicio: una apuesta por su internacionalización en la Educación Superior. Revista Complutense de Educación, 30(1), 1-15. Recuperado de https://revistas.ucm.es/index.php/ RCED/article/view/55443

Zimmerman, B.J. y Shunk, D. H. (2008). Motivation: An essential dimension of self- regulated learning. En D. H. y Zimmerman (Eds.), Motivation and self-regulated learning: Theory, research and applications 1-30. Mahwah: Erlbaum. 